\title{
LC2010: Relativistic Hadron and Particle Physics
}

Light Cone Dynamics is a general Hamiltonian framework, well suited to describe relativistic bound state systems, among them hadrons and nuclei, and their interactions, starting from the first principles of Quantum Chromodynamics.

The various questions relevant to reach this ambitious goal are discussed in a series of workshops held each year under the supervision of the International Light Cone Advisory Committee (ILCAC,Inc).

The 2010 edition of this series took place in Valencia (Spain), between the 14th-18th of June. The Workshop received generous contributions from a variety of sponsors, most notably the Generalitat Valenciana (the local government of the Valencia Community), the Department of Theoretical Physics of the University of Valencia and the University of Valencia through its research fund, The Institute for Particle Physics (IFIC), the Consejo Superior de Investigaciones Científicas (CSIC), the National Center for Particle, Astroparticle, and Nuclear Physics (CEPAN), the European Physical Journal A (EPJA), Hadron Physics2 and the ILCAC.

LC2010 was attended by approximately 80 physicists, and featured a total of 68 presentations (invited talks and posters). It took place at the beautiful Pineda Palace, site of the Universidad Internacional Menéndez Pelayo, in the medieval quarter of the city.

This years the program was extended to incorporate field theory at finite temperature and density, and invited presentations of the most relevant experimental collaborations. Due to the large number of participants, and the high quality of the submitted contributions, parallel sessions were held two out of the five days of the Workshop. It turned out as a very lively conference with extremely interesting presentations and engaging discussions, which showed the healthy state of this particular field of research.

The main topics included: Light front field theory and other non perturbative approaches, relativistic nuclear and hadron structure, non perturbative methods and renormalization theory, AdS/CFT overview and application to strongly interacting systems, recent results in perturbtative QCD, lattice and hamiltonian QCD, relativistic heavy ion physics, GPDs, DVCS and TMDs, the pion transition form factor and the BaBar data.

In addition, S. Brodsky, on the occasion of his seventieth birthday, delivered a special lecture in the Aula Magna of the old University building, sponsored by the European Physical Journal A, reflecting his personal view of Light Cone physics, distilled over the many years 
of pioneering participation in this field.

The Workshop started with a session on light-front field theory and other non perturbative approaches, where some of the most important developments and prominent challenges of non-perturbative Hamiltonian light-front field theory were presented.

The second session was dedicated to relativistic nuclear and hadron structure. Various studies were presented, such as the deuteron electromagnetic form factor using light-front Hamiltonian dynamics, light nuclei, relativistic quantum mechanical descriptions, effective field theory approaches, Regge behavior in exclusive processes, sum rule analysis of the tetraquark, and others.

The third session dealt with non perturbative methods and renormalization theory. Several talks were dedicated to the interpretation of recent lattice results on QCD Green's functions by means of Schwinger-Dyson equations and other non-perturbative approaches.

The fourth session presented and overview on AdS/CFT applied to strongly interacting systems, the remarkable connection between light-front QCD and AdS gravity was discussed, and applications to the light mesons and baryon spectrum and form factors were elaborated.

Session five discussed recent results on perturbtative QCD, with emphasis on calculations of interest for LHC physics, recent developments in the treatment of loops, multiparticle production, and two photon decays at LHC.

Session six was dedicated to lattice QCD. The history and status of high-precision lattice QCD was reviewed, the most important results were summarized, and the limitations of lattice simulations discussed. In addition, the status of QCD calculations on hadronic properties, results regarding the pion wave function, and the gluon structure function in Hamiltonian lattice QCD were presented in various talks.

The physics of the seventh session was new to the workshop series. It dealt with relativistic heavy ion physics and its relation with field theory at high temperature and density. There were experimental presentations discussing ALICE physics, recent results at RHIC and ultra peripheral collisions, as well as theoretical talks on different interesting phenomena that can be extracted from present data, focusing on different theoretical perspectives and future opportunities.

In the eight session overviews on generalized parton distributions, deep inelastic virtual scattering and transverse momentum distribution were given, and the actual experimental situation from the perspective of the last Hermes data was discussed. In addition, various 
theoretical points of view regarding these very interesting developments were presented.

The Conference ended with a particularly lively session on the pion transition form-factor and the BaBar data. The Babar collaboration data in the momentum transfer region of 4 to $40 \mathrm{GeV} 2$ were discussed, and various models and new theoretical approaches for explaining the observed behavior were proposed.

During the Conference dinner, Ben Bakker (chair of the ILCAC) presented awards to this year's Gary McCartor Fellows, Cedric Lorce (University of Mainz), Usha Kulshreshtha (University of Delhi), and Asmita Mukherjee (India Institute of Technology, Mumbay).

We would like to thank all participants for the quality of their contributions, and hope that these Proceedings will serve as a lasting reminder of a most enjoyable and productive Conference.

The local organizing committee

J. Papavassiliou and V. Vento (Chairpersons),

A.C.Aguilar, A. Courtoy, and J.Portolés (Scientific Secretaries). 